RIMS-878

\title{
Topological Lattice Models in Four Dimensions
}

\author{
Hirosi OOGURI 1 \\ Research Institute for Mathematical Sciences \\ Kyoto University, Sakyo-ku, Kyoto 606-01, Japan \\ Dedicated to Professors Huzihiro Araki and Noboru Nakanishi \\ on the occasion of their sixtieth birthdays
}

\begin{abstract}
We define a lattice statistical model on a triangulated manifold in four dimensions associated to a group $G$. When $G=S U(2)$, the statistical weight is constructed from the $15 j$-symbol as well as the $6 j$-symbol for recombination of angular momenta, and the model may be regarded as the four-dimensional version of the Ponzano-Regge model. We show that the partition function of the model is invariant under the Alexander moves of the simplicial complex, thus it depends only on the piecewise linear topology of the manifold. For an orientable manifold, the model is related to the so-called $B F$ model. The $q$-analogue of the model is also constructed, and it is argued that its partition function is invariant under the Alexander moves. It is discussed how to realize the 't Hooft operator in these models associated to a closed surface in four dimensions as well as the Wilson operator associated to a closed loop. Correlation functions of these operators in the $q$ deformed version of the model would define a new type of invariants of knots and links in four dimensions.
\end{abstract}

May, 1992

1 e-mail: ooguri@kekvax.kek.ac.jp, ooguri@jpnyitp.bitnet 
Recently several numerical results have been reported in the three and fourdimensional simplicial quantum gravities concerning their phase structure [1], [2]. Especially in four dimensions, it is suggested in [2] that there is a second order phase transition point indicating the continuum limit of the model. In view of the importance of this suggestion, it is desirable to develop an alternative analytical approach to the dynamical triangulations.

In two dimensions, the matrix model [3] has been found a useful tool in studying the simplicial gravity, and there have been some attempts to extend it to higher dimensions [4]. In this so-called tensor model, one considers an integral over rank-3 tensors rather than matrices, and the perturbative expansion of the integral generates randomly triangulated three-dimensional manifolds. However, in this model, it is found difficult to have control over topologies generated by the random triangulation. This is because the model does not contain the sufficient number of parameters to distinguish different topologies. To solve this problem, Boulatov [5] proposed an improved version of the model defined in terms of functions with three variables on a group or a quantum group rather than the tensors. In his model, the perturbative expansion of the integral is expressed as a sum over triangulated three-manifolds weighted with some topological invariant which depends on the choice of the group $G$. There is a large variety of topological weights corresponding to various choices of groups, and one may hope to gain control in summing over topologies with suitable choice of groups.

The purpose of this paper is two-folded. First we will extend the Boulatov's model to four dimensions and check that its perturbative expansion is expressed as a sum over triangulated four-manifolds. We then show that the weight for the summation is invariant under the Alexander moves [6] [7] of the simplicial complex, and thus depends only on the combinatorially equivalent class of the triangulation. This model could be useful for analytical study of the four-dimensional simplicial gravity. It is known that there is no algorithmical way to classify topologies in four dimensions, but it would still be possible that, for a suitable choice of $G$, the perturbative expansion of the model is dominated by $S^{4}$ or by some restricted set of topologies 2 .

\footnotetext{
2 This seems to be the case in three dimensions [0].
} 
The topological invariants obtained in this way are interesting in their own right, and examining their properties is the second purpose of this paper 3 . In order to solve the quantum gravity in four dimensions, independently of how one defines it, one would need to have a good understanding on four-dimensional geometries. Thus it should be useful to study various geometrical invariants formulated in the language of quantum field theory and statistical mechanics. In the three-dimensional model of Boulatov, if we choose $G$ to be the quantum group $S U_{q}(2)$, the weight in summing over topologies is constructed from the $6 j$-symbols of $S U_{q}(2)$ [9]. The weight then reproduces the three-dimensional topological invariant defined by Turaev and Viro [10], whose $q \rightarrow 1$ limit has been studied many years ago by Ponzano and Regge [11]. It has been shown in [12] [13] that the Turaev-Viro invariant is related to the partition function of the Chern-Simons gauge theory [14] [15] in three dimensions. We shall see that this feature persists in four dimensions. For $q=1$, the four-dimensional model constructed here makes use of the $15 j$-symbols as well as the $6 j$-symbols, and it may be regarded as a four-dimensional version of the Ponzano-Regge model. It is also possible define the $q$-analogue of this model, whose partition function could be regarded as a four-dimensional version of the Turaev-Viro invariant. Although the proof of the topological invariance given here only applies to the case of $q=1$, we present an evidence that it should also hold for when $q$ is a root of unity. For $q=1$, the lattice model is related to the so-called $B F$ model [16] [17] [18. We have not been able to identify a quantum field theory corresponding to the $q$-analogue model, and this task is left for future investigation.

In the three-dimensional Chern-Simons theory, the Wilson operators [19] along closed loops make a basic set of observables and their expectation values are related to the Jones invariants of knots [14]. One may also consider the 't Hooft operator [20], but it is known that the 't Hooft operator is identical to the Wilson operator in the Chern-Simons theory [21]. In four dimensions, on the other hand, it is a surface that makes a non-trivial knot. We study the 't Hooft operator in the $B F$ model, which is associated to $S^{2}$ embedded in four dimensions and differs from the Wilson operator, and discuss how to realize it in the lattice model. Both the 't Hooft operator and the Wilson operator create physical states

3 In this paper, we discuss geometries in the piecewise linear category. If two differential manifolds are diffeomorphic, their smooth triangulations are combinatorially equivalent [8]. Thus an invariant of the piecewise linear topology gives a diffeomorphism invariant of smooth manifolds. In four dimensions, it is not know whether a homeomorphism of two combinatorial manifolds also implies their combinatorial equivalence. This is known to be the case in two and three dimensions. 
on $S^{2} \times S^{1}$ embedded in the four-dimensional manifold $M$. In the case of the 't Hooft operator, $S^{2} \times S^{1}$ is a boundary of $S^{2} \times D^{2}$ embedded in $M$, and its $S^{1}$-part is contractable. On the other hand, for the Wilson operator, $S^{2} \times S^{1}$ is a boundary of $D^{3} \times S^{1}$, and the $S^{1}$-part may link with other operators. Correlation functions of the these operators in the $q$-deformed version of our lattice model would define a new type of invariants of knots and links in four dimensions.

Let us define our model. We start with a real-valued function of four variables $\phi\left(g_{1}, g_{2}, g_{3}, g_{4}\right)$ on $G\left(g_{i} \in G\right)$. For simplicity of notations, we describe the case when $G=S U(2)$, but extensions to other groups should be obvious. If we take $G$ to be a finite group, the model constructed below may be regarded as a four-dimensional version of the Dijkgraaf-Witten model [22]. The function $\phi$ can be expanded in terms of matrix elements $D_{m n}^{j}(g)$ of a spin- $j$ representation as

$$
\phi\left(g_{1}, g_{2}, g_{3}, g_{4}\right)=\sum_{j_{i}, m_{i}, n_{i}} \phi_{m_{1} n_{1} \ldots m_{4} n_{4}}^{j_{1} \ldots j_{4}} D_{m_{1} n_{1}}^{j_{1}}\left(g_{1}\right) \cdots D_{m_{4} n_{4}}^{j_{4}}\left(g_{4}\right)
$$

We then require $\phi$ to be invariant under the right action of $G$,

$$
\phi\left(g_{1} U, g_{2} U, g_{3} U, g_{4} U\right)=\phi\left(g_{1}, g_{2}, g_{3}, g_{4}\right), \quad(U \in G)
$$

This condition is equivalent to

$$
\phi\left(g_{1}, g_{2}, g_{3}, g_{4}\right)=\int d U \phi\left(g_{1} U, g_{2} U, g_{3} U, g_{4} U\right)
$$

where $d U$ is the invariant Haar measure on $G$ normalized as $\int d U=1$. If $G$ is a finite group, the integral is replaced by a summation over group elements divided by the cardinality of the group. With this normalization, integrals of products of $D_{m n}^{j}$ 's become

$$
\begin{aligned}
\int d U \prod_{i=1}^{2} D_{m_{i} n_{i}}^{j_{i}}(U) & =\delta^{j_{1}, j_{2}} g_{m_{1} m_{2}}^{j_{1}} g_{n_{1} n_{2}}^{j_{2}} \\
\int d U \prod_{i=1}^{3} D_{m_{i} n_{i}}^{j_{i}}(U) & =\left(\begin{array}{ccc}
j_{1} & j_{2} & j_{3} \\
m_{1} & m_{2} & m_{3}
\end{array}\right)\left(\begin{array}{ccc}
j_{1} & j_{2} & j_{3} \\
n_{1} & n_{2} & n_{3}
\end{array}\right) \\
\int d U \prod_{i=1}^{4} D_{m_{i} n_{i}}^{j_{i}}(U)= & \sum_{j, m, m^{\prime}, n, n^{\prime}}\left(\begin{array}{ccc}
j_{1} & j_{2} & j \\
m_{1} & m_{2} & m
\end{array}\right) g_{j}^{m m^{\prime}}\left(\begin{array}{ccc}
j & j_{3} & j_{4} \\
m^{\prime} & m_{3} & m_{4}
\end{array}\right) \\
& \left(\begin{array}{ccc}
j_{1} & j_{2} & j \\
n_{1} & n_{2} & n
\end{array}\right) g_{j}^{n n^{\prime}}\left(\begin{array}{ccc}
j & j_{3} & j_{4} \\
n^{\prime} & n_{3} & n_{4}
\end{array}\right) .
\end{aligned}
$$


Here we used the Wigner $3 j$-symbol which is related to the Clebsch-Gordan coefficient as

$$
\left(\begin{array}{ccc}
j_{1} & j_{2} & j_{3} \\
m_{1} & m_{2} & m_{3}
\end{array}\right)=\frac{(-1)^{j_{1}-j_{2}-m_{3}}}{\sqrt{2 j_{3}+1}}\left\langle j_{1} j_{2} m_{1} m_{2} \mid j_{3} m_{3}\right\rangle
$$

and the metric defined by

$$
\begin{aligned}
& g_{m m^{\prime}}^{j}=(-1)^{j-m} \frac{1}{\sqrt{2 j+1}} \delta_{m+m^{\prime}, 0}, \\
& g_{j}^{m m^{\prime}}=(-1)^{j+m} \sqrt{2 j+1} \delta_{m+m^{\prime}, 0} .
\end{aligned}
$$

By substituting (11) into (2) and using the above formulae, we see that $\phi$ is expanded as

$$
\begin{aligned}
\phi\left(g_{1}, g_{2}, g_{3}, g_{4}\right)=\sum & M_{m_{1} m_{2} ; m_{3} m_{4}}^{j_{1} j_{2} ; j ; j_{3} j_{4}} D_{m_{1} n_{1}}^{j_{1}}\left(g_{1}\right) \cdots D_{m_{4} n_{4}}^{j_{4}}\left(g_{4}\right) \\
& g_{j_{1}}^{n_{1} n_{1}^{\prime}} \cdots g_{j_{4}}^{n_{4} n_{4}^{\prime}}\left(\begin{array}{ccc}
j_{1} & j_{2} & j \\
n_{1}^{\prime} & n_{2}^{\prime} & n
\end{array}\right) g_{j}^{n n^{\prime}}\left(\begin{array}{cccc}
j & j_{3} & j_{4} \\
n^{\prime} & n_{3}^{\prime} & n_{4}^{\prime}
\end{array}\right),
\end{aligned}
$$

where

$$
M_{m_{1} m_{2} ; m_{3} m_{4}}^{j_{1} j_{2} ; j j_{3} j_{4}}=\sum_{n, n^{\prime}, n_{i}, n_{i}^{\prime}} \phi_{m_{1} n_{1} \ldots m_{4} n_{4}}^{j_{1} \ldots j_{4}} g_{j_{1}}^{n_{1} n_{1}^{\prime}} \ldots g_{j_{4}}^{n_{1} n_{1}^{\prime}}\left(\begin{array}{ccc}
j_{1} & j_{2} & j \\
n_{1}^{\prime} & n_{2}^{\prime} & n
\end{array}\right) g_{j}^{n n^{\prime}}\left(\begin{array}{ccc}
j & j_{3} & j_{4} \\
n^{\prime} & n_{3}^{\prime} & n_{4}^{\prime}
\end{array}\right) .
$$

There are several conditions that the coefficient $M$ should obey. The real-valuedness of $\phi$ implies

$$
\bar{M}_{m_{1} m_{2} ; m_{3} m_{4}}^{j_{1} j_{2} ; j j_{3} j_{4}}=(-1)^{\sum_{i=1}^{4}\left(j_{i}-m_{i}\right)} M_{-m_{1}-m_{2} ;-m_{3}-m_{4}}^{j_{1} j_{2} ; j ; j_{3} j_{4}}
$$

where we used the hermiticity of $D_{m n}^{j}$

$$
\bar{D}_{m n}^{j}(g)=(-1)^{\sum_{i=1}^{4}\left(j_{i}-m_{i}\right)} D_{-m-n}^{j}(g) .
$$

We also require $\phi\left(g_{1}, g_{2}, g_{3}, g_{4}\right)$ to be invariant under cyclic permutations of any three of its arguments. By the definition (5), this means that the coefficient satisfies

$$
M_{m_{3} m_{1} ; m_{2} m_{4}}^{j_{3} j_{1} ; j ; j_{2} j_{4}}=\sum_{j^{\prime}}(-1)^{j_{1}+j_{2}+j_{3}+j_{4}} \sqrt{(2 j+1)\left(2 j^{\prime}+1\right)}\left\{\begin{array}{lll}
j_{1} & j_{2} & j^{\prime} \\
j_{3} & j_{4} & j
\end{array}\right\} M_{m_{1} m_{2} ; m_{3} m_{4}}^{j_{1} j_{2} ; j^{\prime} ; j_{3} j_{4}}
$$

and similar relations obtained by cyclic permutations of $\left(j_{1}, j_{2}, j_{3}, j_{4}\right)$. Here the symbol $\{\cdots\}$ in the right hand side is the Racah-Wigner $6 j$-symbol describing recombination of three angular momenta. 
Now let us define our action.

$$
\begin{array}{r}
S=\frac{1}{2} \int \prod_{i=1}^{4} d g_{i} \phi^{2}\left(g_{1}, g_{2}, g_{3}, g_{4}\right)+\frac{\lambda}{5 !} \int \prod_{i=1}^{10} d g_{i} \phi\left(g_{1}, g_{2}, g_{3}, g_{4}\right) \phi\left(g_{4}, g_{5}, g_{6}, g_{7}\right) \\
\phi\left(g_{7}, g_{3}, g_{8}, g_{9}\right) \phi\left(g_{9}, g_{6}, g_{2}, g_{10}\right) \phi\left(g_{10}, g_{8}, g_{5}, g_{1}\right)
\end{array}
$$

A four-dimensional simplex consists of five tetrahedra (3-simplexes), ten triangles (2simplexes), ten links (1-simplexes) and five vertices (0-simplexes). If we imagine that the function $\phi\left(g_{1}, g_{2}, g_{3}, g_{4}\right)$ is associated to a tetrahedron with the group elements on its faces, the kinetic term in the above action is seen as overlapping of two tetrahedra while the interaction term represents gluing faces of five tetrahedra to make a 4-simplex. Substituting (4) into the above and using (3), the action can be expressed in terms of $M$ as

$$
\begin{aligned}
S(M)= & \frac{1}{2} \sum_{j, m}\left|M_{m_{1} m_{2}, m_{3} m_{4}}^{j_{1} j_{2}, j_{3} j_{4}, j}\right|^{2} \\
- & \frac{\lambda}{5 !} \sum_{j, m, n}(-1)^{\sum_{i=1}^{10}\left(j_{i}+m_{i}\right)}(-1)^{\sum_{i=1}^{5}\left(l_{i}+n_{i}\right)}\left\{\begin{array}{lllll}
l_{1} & l_{2} & l_{3} & l_{4} & l_{5} \\
j_{1} & j_{2} & j_{3} & j_{4} & j_{5} \\
l_{10} & l_{9} & l_{8} & l_{7} & l_{6}
\end{array}\right\} \\
& M_{m_{2} n_{2} ;-n_{4} m_{3}}^{j_{2} l_{2} ; l_{3} ; l_{4} j_{3}} M_{m_{4} n_{4} ;-n_{6} m_{5}}^{j_{4} l_{4}, l_{5} ; l_{6} j_{5}} M_{-m_{2} n_{6} ;-n_{8} m_{1}}^{j_{2} l_{6} ; l_{7} ; l_{8} j_{1}} M_{-m_{3} n_{8} ;-n_{10}-m_{5}^{\prime}}^{j_{3} l_{8} ; l_{9} ; l_{10} j_{5}} M_{-m_{4} n_{10} ;-n_{2}-m_{1}}^{j_{4} l_{10} ; l_{1} ; l_{2} j_{1}} .
\end{aligned}
$$

Here

$$
\begin{aligned}
\left\{\begin{array}{rllll}
l_{1} & l_{2} & l_{3} & l_{4} & l_{5} \\
j_{1} & j_{2} & j_{3} & j_{4} & j_{5} \\
l_{10} & l_{9} & l_{8} & l_{7} & l_{6}
\end{array}\right\}= & \sum_{m, n}(-1)^{\sum_{i=1}^{10}\left(j_{i}+m_{i}\right)}(-1)^{\sum_{i=1}^{5}\left(l_{i}+n_{i}\right)}\left(\begin{array}{ccc}
j_{1} & l_{1} & l_{2} \\
m_{1} & n_{1} & -n_{2}
\end{array}\right) \\
& \left(\begin{array}{ccc}
j_{2} & l_{2} & l_{3} \\
m_{2} & n_{2} & -n_{3}
\end{array}\right)\left(\begin{array}{ccc}
j_{3} & l_{3} & l_{4} \\
m_{3} & n_{3} & -n_{4}
\end{array}\right)\left(\begin{array}{ccc}
j_{4} & l_{4} & l_{5} \\
m_{4} & n_{4} & -n_{5}
\end{array}\right) \\
& \left(\begin{array}{ccc}
j_{5} & l_{5} & l_{6} \\
m_{5} & n_{5} & -n_{6}
\end{array}\right)\left(\begin{array}{ccc}
j_{2} & l_{6} & l_{7} \\
-m_{2} & n_{6} & -n_{7}
\end{array}\right)\left(\begin{array}{ccc}
j_{1} & l_{7} & l_{8} \\
-m_{1} & n_{7} & -n_{8}
\end{array}\right) \\
& \left(\begin{array}{ccc}
j_{3} & l_{8} & l_{9} \\
-m_{3} & n_{8} & -n_{9}
\end{array}\right)\left(\begin{array}{cccc}
j_{5} & l_{9} & l_{10} \\
-m_{5} & n_{9} & -n_{10}
\end{array}\right)\left(\begin{array}{ccc}
j_{4} & l_{10} & l_{1} \\
-m_{4} & n_{10} & -n_{1}
\end{array}\right)
\end{aligned}
$$

is the $15 j$-symbol of the third kind [23]. It is not difficult to see that the $15 j$-symbol defined in this way can be expressed in terms of the $6 j$-symbols. Although the $15 j$-symbol does not possess the full rotational symmetry of the 4-simplex, the symmetry is recovered in the interaction term of the action thanks to the condition (6) satisfied by $M$.

A partition function of our model is defined as

$$
Z=\int \prod_{j, j_{i}, m_{i}: \sum m_{i}=0} d M_{m_{1} m_{2} ; m_{3} m_{4}}^{j_{1} j_{2} ; j ; j_{3} j_{4}} \exp (-S(M))
$$


As in the case of two [3] and three dimensions [4] [5], we expand this integral in powers of $\lambda$. Each term in this perturbative expansion is constructed from a propagator derived from the kinetic term in the action and a vertex given by the $15 j$-symbol. The non-vanishing components of the propagator are

$$
\begin{aligned}
& \left\langle M_{m_{1} m_{2} ; m_{3} m_{4}}^{j_{1} j_{2} ; j ; j_{3} j_{4}} \bar{M}_{m_{1}^{\prime} m_{2}^{\prime} ; m_{3}^{\prime} m_{4}^{\prime}}^{j_{1} j_{2} ; j ; j_{3} j_{4}}=\frac{1}{9} \prod_{i=1}^{4} \delta^{m_{i}, m_{i}^{\prime}}\right. \\
& \left\langle M_{m_{1} m_{2} ; m_{3} m_{4}}^{j_{1} j_{2} ; j ; j_{3} j_{4}} \bar{M}_{m_{3}^{\prime} m_{1}^{\prime} ; m_{2}^{\prime} m_{4}^{\prime}}^{j_{3} j_{1} ; j^{\prime} ; j_{2} j_{4}}\right\rangle=\frac{1}{9} \prod_{i=1}^{4} \delta_{m_{i}, m_{i}^{\prime}} \sqrt{(2 j+1)\left(2 j^{\prime}+1\right)}\left\{\begin{array}{ccc}
j_{1} & j_{2} & j^{\prime} \\
j_{3} & j_{4} & j
\end{array}\right\} \\
& \left\langle M_{m_{1} m_{2} ; m_{3} m_{4}}^{j_{1} j_{2} ; j ; j_{3} j_{4}} \bar{M}_{m_{2}^{\prime} m_{3}^{\prime} ; m_{1}^{\prime} m_{4}^{\prime}}^{j_{2} j_{3} ; j^{\prime} ; j_{1} j_{4}}\right\rangle=\frac{1}{9} \prod_{i=1}^{4} \delta_{m_{i}, m_{i}^{\prime}} \sqrt{(2 j+1)\left(2 j^{\prime}+1\right)}\left\{\begin{array}{ccc}
j_{1} & j_{2} & j^{\prime} \\
j_{4} & j_{3} & j
\end{array}\right\}
\end{aligned}
$$

and others obtained by the cyclic permutations of $\left(j_{1}, j_{2}, j_{3}, j_{4}\right)$. By combining these elements together, the perturbative expansion of the integral (8) is expressed as

$$
Z=\sum_{C} \frac{1}{N_{\text {sym }}(C)} \lambda^{N_{4}(C)} Z_{C},
$$

where the sum $\sum_{C}$ is over oriented four-dimensional combinatorial manifolds, $N_{\text {sym }}(C)$ is a rank of symmetries of $C$ if any, $N_{4}(C)$ is a number of 4-simplexes in $C$, and $Z_{C}$ is given by a summation which can be written symbolically as

$$
Z_{C}=\sum_{j} \prod_{t: \text { triangles }}\left(2 j_{t}+1\right) \prod_{\text {tetrahedra }}\{6 j\} \prod_{4-\text { simplexes }}\{15 j\} .
$$

To describe the precise content of the summand in the above, it is useful to draw a closed $\phi^{3}$-graph on each 4 -simplex in $C$ as follows. Each 4 -simplex has a topology of a four-dimensional ball and its boundary is covered by five tetrahedra. On the boundary, we can draw a dual $\phi^{4}$-diagram by setting a vertex in each tetrahedron and and by connecting these vertices by links dual to the faces of the tetrahedra. We then split each vertex into two $\phi^{3}$-vertices and add an extra-link between them. There are three different ways to split each $\phi^{4}$-vertex, and we make an arbitrary choice of them at this moment. This defines a closed $\phi^{3}$-graph on each 4 -simplex. By attaching a spin $(j, m)$ on each link and the $3 j$-symbol on each vertex of the $\phi^{3}$-graph $\theta^{-}$and by summing over the magnetic angular

4 Although the $3 j$-symbol is the cyclic symmetric, it changes its sign under odd permutation of the indices. Thus, to be precise, the association of the $3 j$-symbol to the $\phi^{3}$-vertex is unique modulo the sign factor. Since each tetrahedron is shared by two 4 -simplexes, this ambiguity is removed by choosing the same sign convention on the $\phi^{3}$-graphes on the two 4 -simplexes. 
momenta $m$, we obtain a sum of product of the $15 j$-symbol and several $6 j$-symbols [23]. Different choice of the splittings results in different combination of the $6 j$-symbols. This procedure is applied to every 4-simplex in $C$ in such a way that parts of $\phi^{3}$-graphes of two neighboring 4-simplexes match on their common tetrahedron. As a result, we obtain a sum of product of $15 j$-symbols, each of which is associated to a 4 -simplex in $C$, and $6 j$-symbols which depend on how one splits the $\phi^{4}$-vertices. We then perform a sum of this object over all the spins on the extra-links which were added when we split the $\phi^{4}$-vertices. By using the orthonormality of the $6 j$-symbol, one can show that the result of this summation is independent of the choice of splitting of the vertices and depends only on the structure of the complex $C$ and the spins on the links dual to the 2 -simplexes in $C$. This is what we represent by the product $\prod\{6 j\} \prod\{15 j\}$ in the summand of (10).

Now we would like to examine the dependence of $Z_{C}$ on the simplicial complex $C$. In 1930, Alexander [6] defined a set of basic transformations of simplicial complexes and proved that two complexes are combinatorially equivalent if and only if they are connected by a sequence of these transformations. These basic transformations are called the Alexander moves. We would like to know how $Z_{C}$ behaves under the Alexander moves. Recently Gross and Varsted introduced another set of transformations which they showed to be equivalent to the Alexander moves for dimensions less than or equal to four [7], and we shall use them below.

In four dimensions, there are five basic moves [7] [2];

(1) Consider a 4-simplex and add one point at its center. We then draw five 1-simplexes which connect this point to the five vertices of the 4 -simplex. This decomposes the original 4-simplex into five 4-simplexes.

(2) Consider two 4-simplexes sharing one tetrahedron. There remains a pair of vertices which are not shared by the 4-simplexes and we connect them by a 1-simplex. This recombines the two 4-simplexes into three 4-simplexes.

(3) Consider three 4-simplexes sharing one triangle, and suppose that there are three tetrahedron each of which is shared by two of the 4 -simplexes. Then there are three 1simplexes each of which belongs to only one of the 4-simplexes. The three 4-simplexes are recombined into three 4 -simplexes sharing a triangle spanned by these three 1-simplexes. The other two moves are obtained by reversing the moves (1) and (2).

To examine the behavior of $Z_{C}$ under these moves, it is useful to know that the $15 j$ symbols which appear in (10) can be expressed as a sum of product of the $6 j$-symbols [23]. Then the Biedenharn-Elliot identity and the orthonormality of the $6 j$-symbols can be used 
to show that $Z_{C}$ is invariant under these moves upto a multiplicative factors. Rather than showing the detail of the computations, we present here another proof, similar to the one used in [5] in studying the three-dimensional model.

By using the formulae (3) and the definition of the $15 j$-symbol in terms of the $3 j$ symbols, we can rewrite $Z_{C}$ as follows.

$$
Z_{C}=\sum_{j} \sum_{m, n} \prod_{t: \text { triangles }}\left(2 j_{t}+1\right) \prod_{T: t e t r a h e d r a} \int d U_{T} D^{j_{1, T}}\left(U_{T}\right) \cdots D^{j_{4, T}}\left(U_{T}\right)
$$

where each tetrahedron $T$ carries a group element $U_{T}$, and $j_{1, T}, \ldots, j_{4, T}$ are the spins on the links of dual to the four faces of $T$. The matrix elements $D^{j}$ 's are multiplied together around triangles in $C$. Each triangle $t$ is shared by a finite number of tetrahedra $T_{1}^{(t)}, T_{2}^{(t)}, \ldots, T_{n_{t}}^{(t)}$, and we can perform the sum over the spin- $j_{t}$ on the link dual to $t$ using the formula

$$
\sum_{j_{t}}\left(2 j_{t}+1\right) \operatorname{Tr}\left[D^{j_{t}}\left(U_{T_{1}^{(t)}}\right) \cdots D^{j_{t}}\left(U_{T_{n_{t}}^{(t)}}\right)\right]=\delta\left(U_{T_{1}^{(t)}} \cdots U_{T_{n_{t}}^{(t)}}, 1\right) .
$$

If we regard $\left\{U_{T}\right\}_{T: t e t r a h e d r a}$ as giving a set of transition functions of a principal $G$-bundle over the combinatorial manifold, the condition $U_{T_{1}^{(t)}} \cdots U_{T_{n_{t}}^{(t)}}=1$ derived here implies that the holonomy around the triangle $t$ is trivial.

Now we can examine the behavior of $Z_{C}$ under the five basic moves of the simplicial complex. In the first move, the subdivision of a 4 -simplex into five 4 -simplexes creates ten triangles and ten tetrahedra. Correspondingly we introduce ten spin variables $j_{1}, \ldots, j_{10}$ on the triangles and ten group elements $U_{1}, \ldots, U_{10}$ on the tetrahedra, which give transition function of a $G$-bundle in the interior of the original 4-simplex. By summing over $j_{i}$ 's, we obtain ten $\delta$-functions, and they restrict the $G$-bundle in the interior to be flat. Then the ten group elements can be set to 1 by redefining the group elements on the original 4 -simplex. The conditions imposed by these ten $\delta$-functions are not independent and four of them are redundant, giving rise to a factor $\delta(1,1)^{4}$. Thus $Z_{C}$ transforms into $\delta(1,1)^{4} Z_{C}$ under the first move.

If $G$ is a finite group, $\delta(1,1)$ is simply a number of elements in $G$. But it is divergent for a continuous group like $S U(2)$. To regularize this divergence, one may cut off the summation as $j_{i} \leq L$ for some large $L$ as in [11]. The divergent factor is removed from the partition function by multiplying $\delta(1,1)$ for each 0 -simplex and dividing by $\delta(1,1)$ for each 1 -simplex (note that the numbers of the 0 - and 1-simplexes increases by one and five in 
the first move), and we send $L \rightarrow \infty$ after this. In this regularization, the invariance under the first move does not hold at a finite value of $L$ and it is recovered only after taking the limit $L \rightarrow \infty$. Alternatively one may use the $q$-deformed version of the model defined below. When $q$ is a root of unity, $q=e^{2 \pi i /(k+2)}$, the sum over $j$ 's is naturally restricted as $j \leq k / 2$ and the $q$-analogue of $\delta(1,1)$ is given by

$$
\delta(1,1)=\frac{k+2}{2 \sin ^{2}\left(\frac{\pi}{k+2}\right)} .
$$

In the so-called BF model [17] [18 which we will identify as a continuum limit of our lattice model, this divergent factor would correspond to a part of its gauge volume.

In the second move, four triangles are added in the interior of two 4-simplexes and summing over spins on them generates four $\delta$-functions, three of which are independent. Thus $Z_{C}$ transforms into $\delta(1,1) Z_{C}$ under the second move. In this move, the number of the 0 -simplexes does not change while the 1 -simplexes increase by one. Thus the divergence is removed in the same way as in the first move. On the other hand, no redundant $\delta$-function appears in the third move, and $Z_{C}$ remains invariant under this move. The fourth and the fifth moves are the inverses of the first and the second moves, and $Z_{C}$ transforms into $\delta(1,1)^{-4} Z_{C}$ and $\delta(1,1)^{-1} Z_{C}$ respectively. Combining these result, the dependence of $Z_{C}$ on $C$ is expressed as

$$
Z_{C}=\delta(1,1)^{-N_{0}(C)+N_{1}(C)} \chi_{G}(C),
$$

where $N_{0}(C)$ and $N_{1}(C)$ are numbers of 0 - and 1-simplexes in $C$, and $\chi_{G}(C)$ depends only on the combinatorial class of $C$.

It is straightforward to define the $q$-deformed version of the model, by replacing the $15 j$-symbols and the $6 j$-symbols by their $q$-analogues. Since the invariance under the Alexander moves is essentially due to the Biedenharn-Elliot identity and the orthonormality of the $6 j$-symbols, which are also satisfied by their $q$-analogues [9], it is likely that the $q$ analogue model is topological. The detailed analysis will be reported elsewhere.

The expression (11) suggests that, for a compact group $G, Z_{C}$ is related to the partition function of the $B F$ model which is defined for an oriented manifold $M$ as

$$
Z_{B F}=\int[d B, d A] \exp \left(i \int_{M}\langle B, d A+[A, A]\rangle\right),
$$

where $A$ is a connection one-form for a $G$-bundle on the manifold $M, B$ is a $\mathcal{G}$-valued twoform $(\mathcal{G}$ is a Lie algebra of $G$ ) and $\langle$,$\rangle is the invariant bilinear form on \mathcal{G}$. If we integrate over $B$ first, we obtain

$$
Z_{B F}=\int[d A] \delta(d A+[A, A])
$$


and $Z_{B F}$ is given by an integral over flat connections. As we have seen in the above, the set of group elements $\left\{U_{T}\right\}_{T: \text { tetrahedra }}$ in (11) define a principal $G$-bundle on the simplicial manifold, and the sum over $j$ 's restricts the $G$-bundle to be flat. Due to the topological nature of the lattice model, we can take the triangulation to be infinitely fine. When $C$ is a smooth triangulation of $M$, the integral over $U_{T}$ 's in (11) is then identified with the integral over $A$ in (13) in this limit in the sense of the lattice gauge theory. One may also regard the spin- $j$ associated to each 2 -simplex as a discretized version of the two-form $B$.

The action for the $B F$ model has the following gauge symmetries,

$$
\delta A=D \lambda, \quad \delta B=[B, \lambda]+D \omega
$$

where $\lambda$ and $\omega$ are $\mathcal{G}$-valued 0 - and 1 -forms, and $D$ is the covariant derivative with respect to the connection $A$. The Wilson operator associated to a closed loop $\gamma$ is invariant under these gauge transformations and thus gives a physical observable in the theory. One may also try an operator defined by an integral over a closed surface $\Sigma$ in $M$ as $\exp \left(i \int_{\Sigma}\langle\alpha, B\rangle\right)$ for some $\alpha$ in $\mathcal{G}$. Insertion of this operator generates a holonomy $e^{i \alpha}$ around the surface $\Sigma$. This is because the field strength $F$ becomes non-vanishing on $\Sigma$ upon integration over $B$. In the standard Yang-Mills theory, such an operator has been considered by 't Hooft [20], so we call it the 't Hooft operator here. If we take $e^{i \alpha}$ to be in the center of $G$, the operator becomes invariant under the gauge transformations (14).

In order to maintain the gauge symmetries without restricting $e^{i \alpha}$ to be in the center, we consider the following modification to the 't Hooft operator 5 . Let us choose a normal vector field on $\Sigma$ and displace $\Sigma$ slightly in the direction of the vector field to obtain another surface $\Sigma^{\prime}$. The trajectory swept out by $\Sigma$ then defines a three-dimensional submanifold $V_{\Sigma}$ in $M$ bounded by $\Sigma$ and $\Sigma^{\prime}$. This procedure is similar to the framing of the Wilson-line in the three-dimensional Chern-Simons theory [14]. The integration of the $B$ field in $V_{\Sigma}$ restricts $A$ to be flat there, and this induces a flat connection on $\Sigma$ as the boundary value of $A$. Especially when $\Sigma$ is homeomorphic to $S^{2}$, the flat gauge field is trivial and the parallel transport $\Omega(x, y)=P \exp \left(i \int_{y}^{x} A\right)$ is defined globally on $\Sigma$. Thus, in this case, we can define a gauge-invariant operator by

$$
T_{\alpha}(\Sigma)=\int d U \exp \left(i \int_{\Sigma} d^{2} x\left\langle B(x), \Omega(x, y) U \alpha U^{-1} \Omega(y, x)\right\rangle\right) .
$$

5 An operator associated to a surface has also been considered in [24, but it differs from the one constructed here. 
Because of the $U$-integral, this definition of $T_{\alpha}(\Sigma)$ is independent of the choice of a point $y$ on $\Sigma$. It is easy to show that this is invariant under both of the gauge transformations in the above. In the $B F$ model, the diffeomorphism invariance is the consequence of these gauge symmetries. Thus the expectation value of $T_{\alpha}(\Sigma)$ is invariant under homotopy moves of $\Sigma$. If $\Sigma$ is homeomorphic to a torus or a higher-genus surface, the flat gauge field is not necessarily trivial and this construction would need to be modified. In the following, we restrict ourselves to the case when $\Sigma$ is homeomorphic to $S^{2}$.

Now let us discuss how to realize $T_{\alpha}(\Sigma)$ in the lattice model. In the expression (11), each group element $U_{T}$ is associated to a tetrahedron $T$ in the simplicial complex $C$, and each 2 -simplex carries a spin $j_{t}$. For each 2 -simplex $t$, the group elements in the tetrahedra sharing $t$ are multiplied together in the spin- $j_{t}$ representation $D^{j_{t}}$ and the trace of the product is taken. Therefore the holonomy $e^{i \alpha}$ around a given 2-simplex can be imposed by inserting $e^{i \alpha}$ in the trace. In the combinatorial manifold, the surface $\Sigma$ is represented by a collection of 2 -simplexes $\hat{\Sigma}$. Thus we would like to insert $e^{i \alpha}$ in the traces around all the 2 -simplexes on $\hat{\Sigma}$ in such a way that the topological invariance is maintained. This can be done, by discretizing the expression (15), as follows.

Let us choose a subcomplex $\hat{V}_{\Sigma}$ homeomorphic to $S^{2} \times D^{2}\left(D^{2}\right.$ : two-dimensional disc) in $C$ such that the surface $\hat{\Sigma} \simeq S^{2}$ is in the image of its boundary $\left(\simeq S^{2} \times S^{1}\right)$. We first sum over the spin- $j$ 's on the 2 -simplexes in the interior of $\hat{V}_{\Sigma}$. The $G$-bundle in the interior becomes flat, and the group elements in the tetrahedra in the interior can be set to 1 by redefining the group elements on the boundary of $\hat{V}_{\Sigma}$. We then insert $e^{i \alpha}$ on the links dual to the 2-simplexes on $\hat{\Sigma}$. Each 2-simplexes on $\hat{\Sigma}$ is shared by a pair of tetrahedra in $\partial \hat{V}_{\Sigma}$, and the insertion of $e^{i \alpha}$ is made on the links connecting these pairs.

After inserting $e^{i \alpha}$ on $\hat{\Sigma}$, we integrate over the group elements in the tetrahedra in $C$. In the exterior of $\hat{V}_{\Sigma}$, the integral gives the product of the $15 j$-symbols and the $6 j$-symbols as in (10). Therefore the product becomes invariant under the Alexander moves in the exterior of $\hat{V}_{\Sigma}$ after summing over spin- $j$ 's. On the other hand, the integral over the group elements on the boundary $\partial \hat{V}_{\Sigma}$ generates a product of the $3 j$-symbols corresponding to the $\phi^{3}$-graph on $\partial \hat{V}_{\Sigma}$. The product is summed over the magnetic angular momenta on the links with insertions of $e^{i \alpha}$ on the links dual to the 2-simplexes on $\hat{\Sigma}$. This defines a function of spin- $j$ 's on the $\phi^{3}$-graph on $\partial \hat{V}_{\Sigma} \simeq S^{2} \times S^{1}$. By construction, the function depends on the triangulation on $S^{2} \times S^{1}$, but its dependence is cancelled by the contribution from the exterior of $\hat{V}_{\Sigma}$ after summing over the spin- $j$ 's on $\partial \hat{V}_{\Sigma}$. The topological invariance is then maintained. 
In [10] [13], the notion of physical states has been introduced in the topological lattice model in three dimensions. In the three-dimensional model, states on a two-dimensional closed surface are functions of spin- $j$ 's on a $\phi^{3}$-graph dual to a triangulation of the surface, and they are called physical when they transform in a definite way specified in [13] under the Alexander moves of the triangulation. It is possible to introduce a similar notion in the four-dimensional model here. States in the four-dimensional model are functions of spin- $j$ 's on a $\phi^{3}$-graph on a three-dimensional closed manifold, and they are called physical when they transform in such a way that the insertion of the state on the three-manifold embedded in four dimensions does not spoil the topological invariance. We may regard the 't Hooft operator as creating a physical state on $S^{2} \times S^{1}$.

For the 't Hooft operator, $S^{2} \times S^{1}$ is the boundary of $\hat{V}_{\Sigma} \simeq S^{2} \times D^{2}$, and the $S^{1}$-part is contractable in $M$. One may also consider a complementary situation when the $S^{2}$-part is contractable while the $S^{1}$-part may link with other operators. This gives the Wilson operator for a closed loop $\gamma$ which is the image of $S^{1}$. To be more explicit, we consider a subcomplex $\hat{W}_{\gamma}$ homeomorphic to $D^{3} \times S^{1}\left(D^{3}\right.$ : three-dimensional ball) in $C$ such that the loop $\gamma$ travels through tetrahedra $T_{1}, \ldots, T_{n}$ in the interior of $\hat{W}_{\gamma}$. We then make a choice of a spin $j$ and insert the discretized version of the Wilson operator $\operatorname{tr}\left[D^{j}\left(U_{T_{1}}\right) \cdots D^{j}\left(U_{T_{n}}\right)\right]$ in the integrand of (11). By integrating out the group elements in the tetrahedra in $\hat{W}_{\gamma}$ and by summing over the spins on the links in the interior of $\hat{W}_{\gamma}$, we obtain a function of the spins on the boundary of $\hat{W}_{\gamma}$. It is easy to check that the function defines a physical state on $S^{2} \times S^{1}$.

We have constructed the 't Hooft operator associated to a surface $\Sigma$ and the Wilson operator associated to a loop $\gamma$. They both create physical states on $S^{2} \times S^{1}$, so their presence does not spoil the topological invariance of the lattice model. Especially their correlation functions are invariant under ambient isotopy moves of $\Sigma$ and $\gamma$, and thus give invariants of knots and links. It should also be possible to define the 't Hooft operator and the Wilson operator in the $q$-deformed version of the lattice model. Detailed study of these invariants is left for future investigations.

\section{Acknowledgement}

I would like to thank T. Eguchi, K. Higashijima, T. Inami, N. Ishibashi, Y. Yamada, S. Yahikozawa and T. Yoneya for discussions. I would also like to thank the members of the theory group in KEK, where part of this work was done, for their hospitality. 


\section{References}

[1] M.E. Agishtein and A.A. Migdal, Mod. Phys. Lett. A6 (1991) 1863; J. Ambjorn and S. Varsted, Phys. Lett. B226 (1991) 258; D.V. Boulatov and A. Krzywicki, Mod. Phys. Letts A6 (1991) 3005; J. Ambjorn, D. Boulatov, A. Krzywicki and S. Varsted, preprint NBI-HE-91-46 (1991).

[2] M.E. Agishtein and A.A. Migdal, preprint PUPT-1287 (1991), PUPT- (1992); J. Ambjorn and J. Jurkiewicz, preprint NBI-HE-91-47 (1991).

[3] E. Brezin, C. Itzykson, G. Parisi and J.-B. Zuber, Commun. Math. Phys. 59 (1978) 35 .

[4] J. Ambjorn, B. Durhuus and T. Jonsson, Mod. Phys. Lett. A6 (1991) 1133; N. Sasakura, Mod. Phys. Lett. A6 (1991) 2613; M. Douglas and H. Ooguri, unpublished; N. Godfrey and M. Gross, Phys. Rev. D43 (1991) R1749.

[5] D.V. Boulatov, preprint SPhT/92-017 (1992)

[6] J.W. Alexander, Ann. Math. 31 (1930) 292.

[7] M. Gross and S. Varsted, preprint NBI-HE-91-33 (1991)

[8] J.R Munkres, Elementary Differential Topology, Annals of Mathematics Studies 54, (Princeton University Press, 1963).

[9] A.N. Kirillov and N.Yu. Reshetikhin, in Infinite Dimensional Lie Algebras and Groups, edited by V.G. Kac (World Scientific, 1989).

[10] V.G. Turaev and O.Y. Viro, "State Sum Invariants of 3-Manifolds and Quantum 6jSymbols," preprint (1990)

[11] G. Ponzano and T. Regge, in Spectroscopic and Group Theoretical Methods in Physics, ed. F. Bloch (North-Holland, Amsterdam, 1968).

[12] V.G. Turaev, C. R. Acad. Sci. Paris, t. 313, Série I (1991) 395; K. Walker, "On Witten's 3-Manifold Invariants," preprint (1990).

[13] H. Ooguri and N. Sasakura, Mod. Phys. Lett. A6 (1991) 3591; H. Ooguri, preprint RIMS-851 (1991), to be published in Nucl. Phys. B.

[14] E. Witten, Commun. Math. Phys. 121 (1989) 351; Nucl. Phys. B311 (1988/89) 46.

[15] N. Reshetikhin and V.G. Turaev, Invent. Math. 103 (1991) 547.

[16] A.S. Schwarz, Lett. Math. Phys. 2 (1978) 247; Commun. Math. Phys. 67 (1979) 1.

[17] G. Horowitz, Commun. Math. Phys. 125 (1989) 417.

[18] M. Blau and G. Thompson, Ann. Phys. 209 (1991) 129.

[19] K.G. Wilson, Phys. Rev. D16 (1974) 2445.

[20] G. 't Hooft, Nucl. Phys. B138 (1978) 1.

[21] G. Moore and N. Seiberg, Phys. Lett B220 (1989) 422.

[22] R. Dijkgraaf and E. Witten, Commun. Math. Phys. 129 (1990) 393.

[23] A.P. Jucys, I.B. Levinson and V.V. Vanagas, Mathematical Apparatus of the Theory of Angular Momentum (Gordon and Breach, 1964); L.C. Biedenharn, J.D. Louck and 
P.A. Carruthers, The Racah-Wigner Algebra in Quantum Theory, Encyclopedia of Mathematics and its Applications, Volume 9 (Addison-Wesley, 1981).

[24] G.T. Horowitz and M. Srednicki, Commun. Math. Phys. 130 (1990) 83; I. Oda and S. Yahikozawa, Phys. Lett. B 238 (1990) 272. 